\title{
Smoothing of ultrasound images using a new selective average filter
}

Alex F. de Araujo ${ }^{1}$, Christos E. Constantinou ${ }^{2}$ and João Manuel R. S. Tavares ${ }^{1}$

${ }^{1}$ Instituto de Ciência e Inovação em Engenharia Mecânica e Engenharia Industrial, Faculdade de Engenharia, Universidade do Porto, Rua Dr Roberto Frias s/n, 4200-465 - Porto, PORTUGAL, emails: fa.alex@gmail.com, tavares@,fe.up.pt

${ }^{2}$ Department of Urology, School of Medicine Stanford University, Stanford, CA, USA, email: chris.constantinou@alumni.stanford.edu

\section{Corresponding author:}

Prof. João Manuel R. S. Tavares

Faculdade de Engenharia da Universidade do Porto (FEUP)

Departamento de Engenharia Mecânica (DEMec)

Rua Dr. Roberto Frias, s/n, 4200-465 PORTO - PORTUGAL

Tel: +31522 5081487, Fax: +315225081445

Email: tavares@fe.up.pt

Url: www.fe.up.pt/ -tavares 


\title{
Smoothing of ultrasound images using a new selective average filter
}

\begin{abstract}
Ultrasound images are strongly affected by speckle noise making visual and computational analysis of the structures more difficult. Usually, the interference caused by this kind of noise reduces the efficiency of extraction and interpretation of the structural features of interest. In order to overcome this problem, a new method of selective smoothing based on average filtering and the radiation intensity of the image pixels is proposed. The main idea of this new method is to identify the pixels belonging to the borders of the structures of interest in the image, and then apply a reduced smoothing to these pixels, whilst applying more intense smoothing to the remaining pixels. Experimental tests were conducted using synthetic ultrasound images with speckle noisy added and real ultrasound images from the female pelvic cavity. The new smoothing method is able to perform selective smoothing in the input images, enhancing the transitions between the different structures presented. The results achieved are promising, as the evaluation analysis performed shows that the developed method is more efficient in removing speckle noise from the ultrasound images compared to other current methods. This improvement is because it is able to adapt the filtering process according to the image contents, thus avoiding the loss of any relevant structural features in the input images.
\end{abstract}

Keywords: Image Processing; Speckle Noise Smoothing; Selective Filter; Medical imaging; Female Pelvic Cavity. 


\section{Introduction}

According to [Fish, 1990], a sound wave is a mechanical disturbance that crosses an environment resulting in the vibration of the particles presented. The frequency of this vibration is measured in Hertz and when it is higher than $20 \mathrm{KHz}$, it is called ultrasound which cannot be perceived by the human ear. Ultrasound waves, also known as beams, may be longitudinal or transverse. For transverse waves, the direction of disturbance is perpendicular to the propagation, whilst in longitudinal waves the disturbance is in the same direction as the propagation. In both cases, the waves are characterized by wavelength, period, amplitude and frequency, and their transmission velocity depends on the density of the object that receives the ultrasound waves which are produced and detected by a transducer.

In medical ultrasound imaging, a transducer generates a quantity of ultrasound waves at a predefined frequency that are directed onto tissues and organs. The tissues and organs reflect part of these waves and absorb the remaining part. The reflections may be grouped into two main categories according to the reflecting surface: specular reflections occur when the surface hit by the ultrasound waves is larger than the wavelength, and scattering occurs when the surface hit by the ultrasound waves has smaller dimensions than the wavelength used. In the case of scattering, the reflection of the same wave occurs in several directions and therefore only a small part of the wave emitted by the transducer will be received back. Irregular surfaces may also generate the scattering effect [Fish, 1990].

During ultrasonic imaging, a large number of ultrasound wave beams are emitted and a considerable quantity of scattering usually occurs. Besides generating a loss of the signal emitted by the transducer, the scattering of an ultrasonic beam also causes the scattering of other beams due to collisions with other scattered waves. These lost signals are known as echoes and some of them produce artifacts in the resultant image, called "speckle noise", which degrades the spatial resolution and contrast in the image [de Araujo et al., 2014]. These echoes that are displayed during 
the ultrasound examination of organs and other structures captured by the transducer are commonly quantified in shades of grey in which the strongest echoes are represented by higher intensities of grey and the faintest echoes by the lower intensities, i.e. tending to white and black, respectively. Moreover, interpolation and filtering techniques and logarithmic compression may be used to obtain bi-dimensional images, leading to the loss of some signals received by the transducer and therefore, to images of lower quality [Mari and Cachard, 2007]. Despite the usual low quality of the images generated by ultrasonic examinations, this imaging modality is widely adopted for the analysis and diagnosis of organ and tissue dysfunctions because it provides information about the geometry and behaviour of structures in real time without any side effects for the patients [Wu et al., 2010].

As discussed in [Mateo and Fernández-Caballero, 2009], several well-known techniques have been used to smoothing speckle noise in medical images. Among these techniques, some are applied in the spatial domain of the images, and others are used in the frequency domain. The first group, to which the algorithm proposed here belongs, are based on well-known image filtering techniques such as average filtering [Zhang and Wang, 2015], median filtering [Verma et al., 2015] and anisotropic diffusion based filtering [Santos et al., 2013]. The study conducted in [Mateo and Fernández-Caballero, 2009] allows one to conclude that the smoothing algorithms depend heavily on the application. Thus, smoothing algorithms can be incorporated as solutions for noise removal taking into account the type of noise involved, which is strongly related to the image acquisition technique used.

The common artifacts in ultrasound images, such as speckle noise, may give rise to false information of the imaged structures, and so, these artifacts must be removed to avoid any misinterpretations. Based on these facts, a new approach to perform the selective smoothing of images affected by speckle noise is proposed here. This proposal is mainly concerned with medical ultrasound images. The proposed algorithm uses the intensity of the radiation of the image pixels being smoothed in an attempt to select those that potentially belong to the borders of the structures 
of interest allowing, therefore, the adaptive smoothing of the input image by using an average filter with different levels of smoothing.

Usually, an ultrasound image is composed of dissimilar regions of interest affected by speckle noise of different intensities. Thus, applying different smoothing filters or the same smoothing filter but with different smoothing levels in each region of the input images can lead to more competent smoothing of the corrupted images [Thaipanich et al., 2010]. Average filtering was adopted in this study as the smoothing filter since it is a low-pass filter in which high frequencies are filtered and therefore, the relevant details in the internal regions of the imaged structures are preserved [Gonzalez and Woods, 2006].

The rest of this manuscript is organized as follows: related works are presented in the next section. The proposed selective smoothing algorithm is described in Section 3. The experiments and discussion are presented in Section 4. Finally, the conclusions and considerations for future works are pointed out in the last section.

\section{Related works}

Noise removal is a common task in digital image processing and it has been widely studied. This task is included in commonest image pre-processing pipelines and aims to restore the relevant image information corrupted by noise. Hence, an efficient smoothing algorithm is expected to produce an image from the noisy input image that is as similar as possible to the ideal image, i.e. the original image without any noise [Thaipanich et al., 2010].

The presence of noise is common in ultrasound images and interferes severely with, for example, the segmentation, interpretation and classification, i.e. the image analysis, of the anatomical structures [Amirmazlaghani and Amindavar, 2012, Lee et al., 2012]. The main noise in ultrasound images is from the overlapping and deviation of sound waves emitted and received by the ultrasound transducer used. Thus, the study of noise reduction techniques for attenuating the noise 
effects is critical to implement competent computational algorithms designed to improve the quality of the corrupted images. This improvement is particularly crucial to ensure that the segmentation and analysis of the imaged structures can be successfully achieved by computational methods [Narayanan and Wahidabanu, 2009].

In recent years, different computational image processing techniques have been proposed for noise removal, including techniques based on Gaussian filters [Yang et al., 2011, Adams et al., 2009], differential equations [Barcelos et al., 2003, Aubert and Aujol, 2008, Huang et al., 2009, Ghita and Whelan, 2010, Ghita et al., 2012] and multi-resolution processing [Jansen, 2001, Coupé et al., 2012]. Additive and speckle noises are the main types of noise found in ultrasound images. In general, removal of speckle noise is more complex and therefore, speckle noise is commonly treated as additive noise. Hence, several studies on speckle noise removal have used similar approaches to the ones adopted for smoothing images affected by additive noise: for example, Jin and Yang [Jin and Yang, 2011] proposed a new variational model for the removal of speckle noise in ultrasound images based on the model developed by Rudin et al. [Rudin et al., 1992] to remove additive noise. Other variational models have been adopted for the removal of this kind of noise. Aubert and Aujol [Aubert and Aujol, 2008] proposed a new non-convex variational model based on the classic Maximum A Posteriori (MAP) regularization technique to reduce the interference caused by speckle noise in Synthetic-Aperture Radar (SAR) images. In their approach, the speckle model considered that an image affected by speckle noise is a result of its ideal version multiplied by a noise component. Huang and co-authors [Huang et al., 2009] proposed a variational model based on the Total Variation method to overcome the problem of speckle noise removal. The authors reported results similar to those obtained by the method proposed by Aubert and Aujol [Aubert and Aujol, 2008]. These proposals may get good smoothing results, but the parameter that controls the smoothing process must be very accurately defined. However, the setting of this parameter is generally challenging as it strongly depends on the type of image to be smoothed and on the structures it contains. 
Other studies are based on the application of the Wavelet transform for image noise removal [Pizurica et al., 2003, Chang et al., 2000a, Chang et al., 2000b]. In general, these smoothing algorithms can be summarized in three steps. For example, the algorithm proposed by Pizurica et al. [Pizurica et al., 2003] begins by processing the original image using the discrete Wavelet transform, then the desired smoothing operation is performed in the frequency domain and finally, the inverse Wavelet transform is applied to obtain the smoothed image. The advantage of using this kind of approach is that the smoothing of different layers, at different resolutions of the original image, allows the smoothing process to be conducted in such a way that it preserves the specific features in the input image. However, the accuracy and the efficiency of the smoothing depend strongly on the second step of the method and of the type of images to be smoothed.

Approaches based on Gaussian filters are also commonly used in image smoothing [Yang et al., 2011, Adams et al., 2009]. These filters can obtain good results when applied on images with homogeneous regions, but they can lead to excessive loss of information on the borders of structures presented. Such filters are usually developed using a Gaussian function as a convolution kernel, and by recalculating the values of the image pixels based on the convolution of an n-sized window. The intensity of smoothing is controlled by a constant that represents the maximum standard deviation adopted by the Gaussian function used. There are other approaches that also use the Gaussian filter but use various parameters to avoid a high smoothing effect on the borders of the structures in the original image, like the bilateral filter [Tomasi and Manduchi, 1998] and the anisotropic diffusion filter [Perona and Malik, 1990]. The bilateral filter performs a non-linear smoothing of the original image and has been widely used in image processing [Bennett and McMillan, 2005, Zhang and Allebach, 2008]. In addition to considering the neighbouring pixels during the convolution process, as is done by the Gaussian filter, the bilateral filter also takes into account the variation in the intensities of pixels within the convolution window, i.e., it considers the intensity of the spatially close pixels that have similar features in the calculations. So, besides the standard deviation, the bilateral filter has a second parameter that controls the similarity of the 
pixels to be taken into account [Pantelic et al., 2007]. This filter has been studied and tested in medical images, as in [Gupta et al., 2014] where it is used jointly with the ripplet transform to reduce the effect of speckle noise in medical ultrasound images. This filter performs the linear smoothing of the input image, trying to preserve the edges, i.e. the borders, of the structures presented. A disadvantage of this filter is the difficulty to define the values of its parameters and of the number of iterations for the efficient processing of a particular class of input image. An excessive number of iterations, for example, may cause undue loss of image information affecting the quality of possible further processing results, such as segmentation and classification of the structures [Shao et al., 2013].

A smoothing algorithm based on anisotropic diffusion was proposed by Perona and Malik [Perona and Malik, 1990]. The selective smoothing of the input image based on the heat diffusion equation on a surface is the primary feature of this algorithm. The primary characteristic of this algorithm is a delaying of the smoothing effect on the borders and transitions of the structures in the input image, as happens with non-linear heat diffusion on heterogeneous surfaces. Based on the solution proposed by Perona and Malik, other smoothing algorithms have been proposed, such as the Detail Preserving Anisotropic Diffusion (DPAD) algorithm [Aja-Fernandez and Alberola-Lopez, 2006]. Anisotropic diffusion-based filters have shown promise in smoothing medical images, but the noise model involved in the input images must be accurately defined, which is very difficult for ultrasound images due to the great variability and non-linearity of the noise involved.

Average and median filters have been the basis of various image smoothing approaches used in many medical image based applications [Mateo and Fernández-Caballero, 2009]. These filters perform in the image spatial domain and restore the intensity of each pixel according to the intensity of its neighbours and have as their main advantages simplicity and low computational cost [Verma et al., 2015, Zhang and Wang, 2015]. However, the blurring of edges and loss of details can occur due to their linear and non-selective processing [Haseena and Sherikh, 2012]. 
Image smoothing algorithms based on Frost [Frost et al., 1982] and Wiener filters have also been adopted in medical image applications. The first filter attempts to split the input image into components related to homogeneous and heterogeneous regions to perform an adaptive smoothing. On the other hand, smoothing algorithms based on Wiener filters define the smoothing level according to the local intensity variance of the input image, applying a more intense smoothing where the variance is smaller. These techniques preserve the image edges and high-frequency information, but the computational time required relative to linear filters is their main disadvantage [Haseena and Sherikh, 2012].

To assess the performance of image smoothing algorithms, image quality metrics can be used. Various image quality metrics have been suggested to evaluate any undesirable alterations to the original images, like the loss of edge details and structural and visual information. From these, the Edge Preservation Index (EPI), Structural Similarity Information Measurement (SSIM), Visual Image Fidelity (VIF), Universal Image Quality Index (IQI), Peak Signal-to-Noise Ratio (PSNR) and Equivalent Number of Looks (ENL) metrics can be stressed. EPI is commonly used to evaluate how much the edges of structures are preserved in a processed image relatively to a reference image. It can also be used to indicate the visual quality of an image after undergoing some processing step, where higher values suggest a better preservation of the edges [Sattar et al., 1997]. To complement the edges preservation assessment, SSIM can be used. The SSIM algorithm receives two images and compares them, returning a value between 0.0 and 1.0 , where 0.0 indicates that the images are totally different, while high values indicate high similarity between the structures in the images under comparison [Wang et al., 2004].

On the other hand, there are metrics used to assess the visual quality of processed images, comparing them against an image taken as a reference. In this class, the VIF and IQI metrics can be stressed, where the former can be used to compare the distortion of a processed image, returning values greater than 0 (zero), with values close to 0 indicating that the image has undergone a major distortion. On the other hand, these metrics can identify when an image has not been distorted by 
the processing process, leading to a value equal to 1.0 (one) and a positive feature of VIF, while values above 1.0 suggest that the processed image has a better visual appearance than the reference image [Sheikh and Bovik, 2006]. The latter metric, the IQI metric was proposed to assess the distortion in an image taking into account the loss of correlation between image blocks as well as luminance and contrast distortions. This metric performs a local analysis in each image block, computing the final metric value by averaging all local metric values. The values achieved by this metric range from -1.0 to 1.0 , where the value 1.0 indicates that the images are identical and -1.0 that they are very unalike [Wang and Bovik, 2002].

To analyse the error caused by an image processing algorithm, the PSNR metric can be used. This metric is one of the best-known image quality metrics based on error that exists in the literature. The PSNR attempts to assess the relationship between the highest possible strength of a signal (in the case of an image, its highest intensity value) and its strength affected by noise in a logarithmic scale on base 10 (decibel). The higher the resultant value, the more similar are the two images under comparison [Dash et al., 2011, de Araujo et al., 2014].

To complement the aforementioned image quality metrics, the ENL metric can be used to statistically assess whether an image region is homogeneous or not, without using another image as reference [Anfinsen et al., 2009]. The higher the resulting value, the more homogeneous the analysed region is.

\section{Proposed Method}

In the medical imaging field, there are two imaging modalities commonly used to produce images of structures in the human body: magnetic resonance imaging [Ma et al., 2010] and computed tomography imaging [Brankov et al., 2004]. These modalities produce images with better resolution than ultrasound imaging which, however, is also widely used. Ultrasound imaging has some specific advantages, such as non-invasive examinations, which means that it can be used on any 
patient, even if he/she has metal implants, is pregnant or uses heart devices. Another attractive point of the ultrasound imaging is its low financial cost, which contributes to a larger number of laboratories offering this type of medical exam. In addition, through ultrasound imaging, the movement and/or deformation of structures can be acquired enabling, for example, functional studies in real-time [Narayanan and Wahidabanu, 2009].

On the other hand, ultrasound imaging is more affected by noise, particularly by speckle noise, which is a disadvantage compared to other medical imaging modalities. The interference caused by speckle noise corrupts the images produced and consequently, the resolution of the images is hampered, hindering the extraction of high-level information of the structures. Thus, an analysis of these images by a specialist may be compromised; also the subsequent steps of computational image processing and analysis may be affected. Therefore, taking into consideration the advantages of the ultrasound imaging modality, speckle noise deserves attention and considerable work has already been done. However, it is stills a problem to be effectively overcome. As emphasized in [Narayanan and Wahidabanu, 2009], the development of efficient algorithms for ultrasound image smoothing demands taking into account factors and requirements of the desired application, such as the noise model involved in the images, the nature of the features of interest, and possibly a priori knowledge about the set of images and features to be handled. In the literature, some of the proposed algorithms have presented advantages for specific applications and disadvantages for others. The literature review performed here shows that speckle noise is non linear and depends highly on the parameters of the signal-generating source or on the type of images and structures involved [Misra and Lim, 2015, Raj and Venkateswarlu, 2012]. Consequently, it becomes necessary to adapt existing algorithms or develop new ones to tackle the speckle noise for specific applications, such as reducing this type of noise in ultrasound images of the female pelvic cavity to facilitate and improve, for example, the segmentation of the organs present in these images by further computational methods. 
A priori information that was important in the development of the smoothing method proposed in this study is the fact that an ultrasound image is usually composed of regions affected in different ways by the speckle noise [Thaipanich et al., 2010]. For example, in regions composed of low frequencies, the interference caused by speckle noise may be perceived more easily. However, because of their homogeneity, the smoothing performed in these regions may be stronger than the smoothing on the borders of these regions [Barcelos and Pires, 2009].

In medical ultrasound images, the presence and interference of speckle noise are lower in smooth organs that usually store a large amount of liquid, such as the bladder because organs filled with liquid absorb a large part of the beams, which avoids the spreading of the ultrasound beams; however, in regions that present harder structures, i.e. bones, the presence of noise is higher because hard structures reflect ultrasonic beams directly back to the transducer causing speckle noise. Transition regions between different structures of an image must be preserved in order not to affect the results of the subsequent steps of the computational processing [Barcelos and Pires, 2009]. In line with this, the concept of the selective filter was adopted in an attempt to smooth medical ultrasound images, particularly of the female pelvic cavity, in a more efficient way. Hence, the proposed method performs a local smoothing of the input image; in other words, it treats each pixel of the image by taking into account the group enclosed by its closest neighbours. As such, the main contribution of this work is the proposal of a new selection criterion for the selective filtering of medical ultrasound images. This work has been mainly based on images of the female pelvic cavity. This new method uses the intensity of the radiation associated to the image pixels being smoothed in an attempt to select the ones that potentially belong to the borders of the structures of interest, allowing, therefore, adaptable smoothing to be performed by an average filter with different smoothing intensities. The average filtering was adopted because it is a low-pass filter, in which high frequencies are filtered, it has an affordable computational cost and permits the intensity of the smoothing to be varied by modifying the size of its convolution window [Gonzalez and Woods, 2006]. 


\subsection{Selective criteria for image smoothing}

The intensity of radiation emitted by a point is the energy flow that it emits per time unit. An interesting property of radiation is that it undergoes changes as it moves away from the point of emission [Incropera, 2006]. Assuming a barrier-free environment, the trend is that radiation reduces in a linear manner. Thus, radiation intensity of a pixel of a grey-scale image can be calculated as:

$R=\frac{P}{A}$

where $R$ represents the intensity of radiation, $P$ represents the power and $A$ represents the area of the region affected by the radiation. Considering the radiation spreading along a circular area and the radiation power as the grey-level of the pixel $i$ of the image $I$, Equation 1 can be rewritten as:

$R_{i}=\frac{I_{i}}{4 \pi r^{2}}$

where $r$ is the radius of the circular area affected by the radiation from the pixel $i$. The radiations $R_{i}$ were adopted to identify which pixels of the input images should be less affected by the smoothing process in the proposed smoothing method.

In order to decide the probability of a pixel to be a noisy one, the radiation intensity received by a pixel $i$ is calculated by adding up all the influences caused by its neighbouring pixels and taking into account the parameter $T_{i}$ that is calculated for each pixel $I$ as discussed in the next section. Equation 2 is rewritten as: 


$$
R_{i}=\sum_{i=1}^{\frac{w}{2}}\left[\left(\frac{I_{i}}{4 \pi r^{2}}\right)-T_{i}\right]
$$

Thus, the radiation intensity $R_{i}$ is calculated considering a circular radiation area with pixel $i$ as its centre and with maximum radius equal to $\frac{w}{2}$ pixels, where $w$ is the width of the original image. The radius value was defined from tests performed during this work that allowed us to conclude that a radius greater than $\frac{w}{2}$ does not lead to different results. Thus, it is unnecessary to compute the radiation emitted from pixels that are very distant from pixel $i$ since this radiation is so low that it can be disregarded. Additionally, only the radiation emitted by neighbouring pixels that belongs to the row, the column or the diagonals that include the pixel $i$ are considered. Hence, the influence of the remainder pixels is discarded in order to not affect the pixel $i$ in the vertical and horizontal directions, which also reduces the associated computational cost.

After calculating the radiation that affects each pixel of the original image, the method attempts to estimate the ones that have higher probability of being noisy, so that they may undergo a stronger smoothing and so that details of the regions of interesting are not affected by the smoothing process, particularly areas representing structural borders.

The experimental evaluation of the method developed in this work demonstrated that a pixel strongly influenced by the radiation of neighbouring pixels has a higher probability of being affected by noise or of belonging to a homogeneous region. Thus, these pixels undergo a stronger smoothing using an average filter with a large sized convolution window (equal to $25 \times 25$ in the tests performed and described in the next section), in order to restore their intensity to a level similar to that of their neighbours. 


\subsection{New selective average filter method}

The proposed smoothing method is based on a selective average filter as depicted by the flowchart in Figure 1. The method uses the average filter to remove the noise that is present in the original image, automatically defining the smoothing level of the filter, i.e. the size of the convolution window, in accordance with the value of the accumulated radiation that the pixel under smoothing receives from the neighbouring pixels. This adaptive size of the convolution window for the average filter is the main novelty and interesting feature of the proposed method.

$<$ Figure 1 should be inserted around here $>$

The proposed methodology starts by smoothing the input image using an average filter with a $3 \times 3$ convolution window. Hence, the smoothing performed in this first step is of low intensity in order to avoid any losses of important details of the structures. The $3 \times 3$ window was chosen because it corresponds to the narrowest window that can be used with the average filter employed here. To avoid excessive changes in the grey-level of the pixels, a parameter referred to as the moderator term $\left(T_{i}\right)$ was included in Equation 3 to prevent any abrupt change in the pixel value relative to its original value. This parameter is calculated for each neighbouring pixel $j$ of the pixel $i$ as:

$$
T_{i}=I_{i}-I_{j},
$$

where $I_{j}$ is the grey-level value of the emitting pixel $j$, and $I_{i}$ is the original grey-level value of the pixel $i$. Then, the radiation received by each pixel $i$ of the input image is calculated using Equation 3, calculating the probability of the pixel to be affected by noise or not. Then, the intensity of each pixel considered as affected by noise is computed using the average filter with a stronger smoothing 
effect using a larger convolution window. The remaining pixels are recalculated using the average filter with a weaker smoothing effect using a smaller convolution window.

\section{Experimental Results}

To verify the accuracy and efficiency of the developed method, experimental tests were conducted using synthetic images, which were obtained from noise-free synthetic images with the addition of: (1) artificial speckle noise varying from 0.1 to 0.3 and (2) ultrasound effects simulated by the Field II software [Jensen, 1996, Jensen and Svendsen, 1992] adopting a linear array B-mode scanning option, and real medical images obtained by ultrasound imaging. The original noise-free synthetic images were used to allow the statistical comparison of the smoothing results.

To compare and analyse the results obtained, the following commonly adopted smoothing methods were also applied to the test images: the smoothing algorithm based on the anisotropic diffusion approach proposed by Perona and Malik [Perona and Malik, 1990], adopting 15 iterations, integration constant equal to $1 / 7$ and gradient modulus threshold equal to 30; the Detail Preserving Anisotropic Diffusion (DPAD) algorithm [Aja-Fernandez and Alberola-Lopez, 2006, Yu and Acton, 2002], with 100 iterations and the size of time step in each iteration as 0.2 ; a $2 \mathrm{D}$ bilateral filter, with 3 as the size of the bilateral Gaussian filter window, spatial domain standard deviation equal to 3 and intensity-domain standard deviation equal to 0.3 [Tomasi and Manduchi, 1998, Yang et al., 2015, Anantrasirichai et al., 2014]; adaptive Frost filtering [Frost et al., 1982]; average and median filtering [Gonzalez and Woods, 2006] using a convolution window of size equal to 3 to avoid excessive loss of details; and Wiener filter with a mask of 5x5 [Naimi et al., 2015]. The parameters adopted throughout the tests with these filters were set based on the recommended values found in the respective references. Moreover, these filters were chosen because they have been commonly used for smoothing medical images. 


\subsection{Synthetic images}

In this section, the results of smoothing the synthetic images with noise obtained from (1) adding artificial speckle noise and (2) using the Field II simulation software are presented and discussed. A set of 20 synthetic images was used to test the proposed method against the other smoothing methods of the literature under comparison. This set of images was composed of 7 images affected by artificial speckle noise with a variance of $0.1,7$ images affected by speckle noise with a variance of 0.3 , and 6 images obtained using Field II.

The first test was conducted using the synthetic images obtained by adding artificial speckle noise to the noisy-free original images. Figure 2 shows three images in the top row: original image in the left column, the resultant image after adding speckle noisy with variance equal to 0.3 in the centre and, an image composed by overlapping the original image with correspondent blocks of the resultant smoothed images by applying the: (A) median filter, (B) average filter, (C) Perona and Malik Anisotropic diffusion filter, (D) proposed selective average filter, (E) DPAD method, (F) 2D Bilateral filter, (G) Frost filter and (H) Wiener filter; and the completely smoothed images are shown in the next rows of the Figure. This Figure clearly shows the superior smoothing results obtained by the DPAD method (E), Wiener filter (H) and the proposed method (D).

$<$ Figure 2 should be inserted around here $>$

The chart presented in Figure 3 statistically complements the visual analysis of the smoothed images presented in Figure 2. The data in the chart indicate that the new method developed here restored the image affected by the artificial speckle noise with the lowest error, as it had the highest value for the PSNR metric. At the same time, the structures and their respective edges were more clearly preserved by the proposed smoothing method, as is evidenced by the highest values obtained for the MSSIM and EPI metrics, respectively. The IQI and VIF metrics also had the highest values for the proposed method, indicating the superior visual quality of the smoothed image. The DPAD 
and Wiener filters also produced smoothed images with good quality, as can be confirmed from the values obtained for the image quality metrics used.

$<$ Figure 3 should be inserted around here $>$

The results obtained by the smoothing filters under comparison of a synthetic ultrasound image built using the Field II simulation software in a synthetic image are shown in Figure 4. The original synthetic image used is in the top row on the left, and in the same row in the centre is the image after the Field II simulation software was applied on the original image. The original image overlapped with the corresponding blocks of the images obtained by the smoothing methods under comparison is shown in the right column of the same row, and the completely smoothed images are shown in the next rows of the Figure $(\mathrm{A}-\mathrm{H})$.

The bar chart in Figure 5 shows that the proposed method was able to produce a smoothed image with less error (highest PSNR value), with better edge preservation and higher value as to the structural information contained (highest EPI and MSSIM values, respectively), compared to the other methods under comparison.

$<$ Figures 4 and 5 should be inserted around here $>$

The Equivalent Number of Looks (ENL) [Anfinsen et al., 2009] obtained for the images shown in Figures 2 and 4 are indicated in Table 1. The ENL values indicate the level of homogenization of the regions identified by the red rectangles drawn in the central top images of Figures 2 and 4 . These regions were manually defined in order to include only one structure. The values in Table 1 demonstrate that the ENL of the regions under analysis in the images smoothed by the anisotropic diffusion filter and the developed method are considerably higher than the remaining values. This shows that these two methods have a greater capacity to restore the homogeneous regions affected 
by synthetic speckle noise than the other methods. Also, Table 1 demonstrates that the filter based on anisotropic diffusion obtained the highest ENL for the image in Figure 4, which can be explained due to the capacity of this filter for homogenization when a high number of iterations are performed. However, the loss of border details, as showed by the MSSIM and EPI metrics (Figures 3 and 5), is a negative aspect of this filter.

Analysing Figure 6, one can visually confirm that the proposed method has another interesting feature, which is its ability to restore the regions damaged by speckle noise, preserving the edges presented in the input image efficiently. This Figure shows that the region smoothed by the proposed method (D) is very similar to the same noisy-free region. The DPAD (E) and the Frost (G) filters also produced good results, but some artifacts were not completely eliminated and were still visible in the smoothed regions.

$<$ Figure 6 should be inserted around here $>$

$<$ Table 1 should be inserted around here $>$

Therefore, the smoothing results obtained from the synthetic images show that the proposed smoothing method can adequately preserve the information of the structures in the original images affected by speckle noise, while effectively smoothing their homogeneous regions, and is more efficient than the other methods under comparison.

In some cases, the proposed method did not have the same ability as the DPAD method to maintain the visual appearance of the homogeneous regions, but it preserved the transitions between the different structures presented in the image more clearly, as seen in Figure 4. Therefore, it can be concluded that the new smoothing method proposed here can, in general, produce good results, as is confirmed by the chart in Figure 7, which represents the average values of the image quality metrics used for all 20 synthetic test images. In fact, this chart confirms the superior performance of the 
proposed selective average smoothing method. Finally, considering the importance of both the quality of the structural information of the smoothed images and the preservation of edge details, the proposed algorithm is shown to be both efficient and very promising for use in the preprocessing of ultrasound images that will be further analysed visually or by computational means.

$<$ Figure 7 should be inserted around here $>$

\subsection{Real ultrasound images}

Similar to the tests conducted using synthetic images, the same smoothing methods were applied to 4325 real ultrasound images using the same filter parameters used for the synthetic images. These images were extracted from three real ultrasound videos of the pelvic cavity and from one real ultrasound video of the carotid artery. The smoothing results obtained for one image randomly selected from each image sequence, which are shown in Figure 8, are presented in Figure 9. Each row of this Figure shows the results of the smoothing obtained for each of the real images tested by the: (A) median filter, (B) average filter, (C) Perona and Malik Anisotropic diffusion filter, (D) proposed selective average filter, (E) DPAD method, (F) 2D Bilateral filter, (G) Frost filter and (H) Wiener filter.

$<$ Figures 8 and 9 should be inserted around here $>$

A visual analysis of the smoothed real images (Figure 9) demonstrates that the DPAD, and the proposed method are more efficient in preserving the transitions between the structures in the original images. In addition the values shown in Table 2 demonstrate that the ENL of the regions of the smoothed images (Figure 9) identified by red rectangles in the corresponding images of Figure 8, are higher for the proposed method, indicating its superior capacity to reduce the noise 
interference in homogeneous regions. This behaviour was also found when all images of the real ultrasound image sequences were taken into account; with the proposed method always achieving the highest average ENL value, Table 2.

$<$ Table 2 should be inserted around here $>$

The analysis of the results obtained based on the PSNR, EPI, VIF, MSSIM, IQI and ENL image quality metrics for the synthetic images as well as the ENL for the real images, allows us to conclude that the proposed method was able to smooth the ultrasound images more efficiently than the current methods that it was compared against.

The main objective of this work was to develop a computational method specifically to smooth ultrasound images. As shown by the experimental analysis undertaken in this work, the proposed method has achieved this objective successfully. To complement the statistical analysis preformed and to introduce the direction for a possible future research, a preliminary analysis of the positive effect caused by the smoothing of real ultrasound images by the proposed method is demonstrated in Figure 10. This Figure presents two real ultrasound images randomly selected from a bladder exam [Peng et al., 2006], and used to demonstrate the positive contribution of the proposed smoothing method in achieving segmentation results of better quality.

$<$ Figure 10 should be inserted around here $>$

A region of the urethra was manually selected in each image of Figure 10 taking into account that it should include part of the anterior and posterior edges of the urethra. The resolution and contrast of these parts were greatly affected by speckle noise. Then, the original and smoothed region images were segmented using the Otsu's thresholding method. The resultant segmented images also presented in Figure 10, show that the segmentations obtained from the images smoothed by the 
proposed method are of superior quality, with more regular edges and with less over-segmented regions, compared to the segmentations obtained from the original images.

\section{Conclusion}

In this paper, a new method was proposed for smoothing ultrasound images that are commonly affected by speckle noise. The method uses an average filter that performs a selective smoothing of the original image based on the analysis of the radiation of each pixel. The experimental testing of the proposed method was divided into two steps. In the first one, synthetic images were used to verify the efficiency and accuracy of the proposed method in preserving the structural information and edge details. In the second step, real medical ultrasound images of the pelvic cavity and the carotid artery were used to analyse the efficiency of the proposed method in speckle noise interference reduction in these kinds of images.

The excessive presence of speckle noise in ultrasound images may affect the analysis of the imaged structures, which makes their posterior analysis, such as their computational segmentation and classification, more difficult. The main contribution of this research was to present a new smoothing method suitable for ultrasound images and able to reduce the interferences caused by speckle noise. The proposed method preserves the transitions between the different structures in the input images while making the regions with small intensity variations more homogeneous. Compared to the current smoothing methods, the best performance found for edge preservation, structural information maintenance and minor error rate was the proposed method. Thus these results qualify it to smooth ultrasound images, particularly when the images are intended to be segmented by computational algorithms.

The selective smoothing method proposed has presented relevant and promising results, as was showed by the analysis undertaken based on different image quality metrics, which indicated the better performance of the proposed method compared to other methods of the same category 
commonly used. However, in some images it was noted that the IQI and VIF metrics indicated a better performance of other methods in terms of the visual quality of the smoothed images, particularly highlighting the DPAD and the 2D Bilateral filters. Thus, the smoothing method proposed in this work may not be the best option for applications where the maintenance of the visual appearance is the main requisite. The solution developed during this work is specially indicated to be used in areas where the existence of speckle noise seriously affects the performance of computational algorithms of image analysis, like segmentation and classification algorithms. Examples of these areas, are those based on different medical imaging modalities and of georeferencing that uses Synthetic Aperture Radar (SAR) images.

We can also explore the possibility of using the proposed methodology with other smoothing filters rather than the average filter used here, like the Gaussian filter, particularly in cases where the images are also strongly affected by additive noise. Another future research that can be developed from the work presented is to apply the proposed smoothing method to the Wavelet layers of the original images leading, therefore, to a multi-resolution smoothing method, making it even more selective, since the processing of Wavelet layers will allow the smoothing of different bands of frequency independently.

\section{Acknowledgments:}

The first author would like to thank "Fundação para a Ciência e a Tecnologia (FCT)", in Portugal, for his PhD scholarship with reference SFRH/BD/61983/2009.

This work was partially developed within the scope of the project "A novel framework for Supervised Mobile Assessment and Risk Triage of Skin Lesions via Non-invasive Screening”, with reference PTDC/BBB-BMD/3088/2012, supported by FCT.

Authors gratefully acknowledge the funding of Project NORTE-01-0145-FEDER-000022 - SciTech

- Science and Technology for Competitive and Sustainable Industries, co-financed by Programa 
“Operacional Regional do Norte (NORTE2020)", through “Fundo Europeu de Desenvolvimento Regional (FEDER)".

\section{References}

[Adams et al., 2009] Adams, A., Gelfand, N., Dolson, J., and Levoy, M. (2009). Gaussian kd-trees for fast high-dimensional filtering. ACM Transacyions on Graphics, 28(3):21:1-21:12.

[Aja-Fernandez and Alberola-Lopez, 2006] Aja-Fernandez, S. and Alberola-Lopez, C. (2006). On the estimation of the coefficient of variation for anisotropic diffusion speckle filtering. IEEE Transactions on Image Processing, 15(9):2694-2701.

[Amirmazlaghani and Amindavar, 2012] Amirmazlaghani, M. and Amindavar, H. (2012). Wavelet domain Bayesian processor for speckle removal in medical ultrasound images. IET Image Processing, 6(8):580-588.

[Anantrasirichai et al., 2014] Anantrasirichai, N., Nicholson, L., Morgan, J. E., Erchova, I., Mortlock, K., North, R. V., Albon, J., and Achim, A. (2014). Adaptive-weighted bilateral filtering and other pre-processing techniques for optical coherence tomography. Computerized Medical Imaging and Graphics, 38(6):526.539.

[Anfinsen et al., 2009] Anfinsen, S., Doulgeris, A., and Eltoft, T. (2009). Estimation of the equivalent number of looks in polarimetric synthetic aperture radar imagery. Geoscience and Remote Sensing, IEEE Transactions on, 47(11):3795-3809.

[Aubert and Aujol, 2008] Aubert, G. and Aujol, J.-F. (2008). A variational approach to removing multiplicative noise. SIAM Journal of Applied Mathematics, 68(4):925-946.

[Barcelos et al., 2003] Barcelos, C. A. Z., Boaventura, M., and Jr., E. C. S. (2003). A wellbalanced flow equation for noise removal and edge detection. IEEE Transactions on Image Processing, 12(7):751-763.

[Barcelos and Pires, 2009] Barcelos, C. A. Z. and Pires, V. B. (2009). An automatic based nonlinear diffusion equations scheme for skin lesion segmentation. Applied Mathematics and Computation, 215(1):251-261.

[Bennett and McMillan, 2005] Bennett, E. P. and McMillan, L. (2005). Video enhancement using per-pixel virtual exposures. ACM Transactions on Graphics, 24(3):845-852. 
[Brankov et al., 2004]Brankov, J. G., Yang, Y., and Wernick", M. N. (2004). Tomographic image reconstruction based on a content-adaptive mesh model. IEEE Transactions on Medical Imaging, 23(2):202-212.

[Chang et al., 2000a] Chang, S. G., Member, S., Yu, B., Member, S., and Vetterli, M. (2000). Spatially adaptive wavelet thresholding with context modeling for image denoising. IEEE Transactions on Image Processing, 9:1522-1531.

[Chang et al., 2000b] Chang, S. G., Yu, B., and Vetterli, M. (2000). Adaptive wavelet thresholding for image denoising and compression. IEEE Transactions on Image Processing, 9(9):1532-1546.

[Coupé et al., 2012] Coupé, P., Manjón, J., Robles, M., and Collins, D. (2012). Adaptive multiresolution non-local means filter for three-dimensional magnetic resonance image denoising. IET Image Processing, 6(10):558-568.

[Dash et al., 2011] Dash, R., Sa, P. K., and Majhi, B. (2011). Restoration of images corrupted with blur and impulse noise. In Proceedings the International Conference on Communication, Computing \& Security, 1:377-382.

[de Araujo et al., 2014] de Araujo, A. F., Constantinou, C. E., and Tavares, J. M. R. (2014). New artificial life model for image enhancement. Expert Systems with Applications, 41(13):58925906.

[Fish, 1990] Fish, P. (1990). Physics and Instrumentation of Diagnostic Medical Ultrasound. Wiley, 1 edition.

[Frost et al., 1982] Frost, V. S., Stiles, J. A., Shanmugan, K., and Holtzman, J. (1982). A model for radar images and its application to adaptive digital filtering of multiplicative noise. Pattern Analysis and Machine Intelligence, IEEE Transactions on, 4(2):157-166.

[Ghita et al., 2012] Ghita, O., Ilea, D., and Whelan, P. (2012). Adaptive noise removal approach for restoration of digital images corrupted by multimodal noise. IET Image Processing, 6(12):11481160.

[Ghita and Whelan, 2010] Ghita, O. and Whelan, P. F. (2010). A new gvf-based image enhancement formulation for use in the presence of mixed noise. Pattern Recognition, 43(8):26462658.

[Gonzalez and Woods, 2006] Gonzalez, R. C. and Woods, R. E. (2006). Digital Image Processing (3rd Edition). Prentice-Hall, Inc., Upper Saddle River, NJ, USA. 
[Gupta et al., 2014] Gupta, D., Anand, R., and Tyagi, B. (2014). Ripplet domain non-linear filtering for speckle reduction in ultrasound medical images. Biomedical Signal Processing and Control, 10:79 -91.

[Haseena and Sherikh, 2012] Haseena, K. A. and Sherikh, K. K. (2012). A view on ultrasonogram denoising techniques, Trends in Innovative Computing 2012 - Information Retrieval and Data Mining, 1:18-23.

[Huang et al., 2009] Huang, Y.-M., Ng, M. K., and Wen, Y.-W. (2009). A new total variation method for multiplicative noise removal. SIAM Journal on Imaging Sciences, 2(1):20-40.

[Incropera, 2006] Incropera, F. P. (2006). Fundamentals of Heat and Mass Transfer. John Wiley \& Sons.

[Jansen, 2001]Jansen, M. (2001). Noise Reduction by Wavelet Thresholding. Lecture Notes in Statistics, Volume 161, Springer, pages 81-100.

[Jensen, 1996]Jensen, J. (1996). Field: A program for simulating ultrasound systems. Medical \& Biological Engineering \& Computing, 34(Suplement 1):351-53.

[Jensen and Svendsen, 1992] Jensen, J. and Svendsen, N. B. (1992). Calculation of pressure fields from arbitrarily shaped, apodized, and excited ultrasound transducers. IEEE Transactions on Ultrasonics, Ferroelectrics and Frequency Control, 39(1):262-267.

[Jin and Yang, 2011] Jin, Z. and Yang, X. (2011). A variational model to remove the multiplicative noise in ultrasound images. Journal of Mathematical Imaging and Vision, 39(1):62-74.

[Lee et al., 2012] Lee, M.-S., Yen, C.-L., and Ueng, S.-K. (2012). Speckle reduction with edges preservation for ultrasound images: using function spaces approach. IET Image Processing, 6:813$821(8)$.

[Ma et al., 2010] Ma, Z., Jorge, R. N. M., and Tavares, J. M. R. S. (2010). A shape guided c-v model to segment the levator ani muscle in axial magnetic resonance images. Medical Engineering \& Physics, 32(7):766-74.

[Mari and Cachard, 2007] Mari, J. and Cachard, C. (2007). Acquire real-time rf digital ultrasound data from a commercial scanner. Electronic Journal "Technical Acoustics", http://www.ejta.org, 2007, 3.

[Mateo and Fernández-Caballero, 2009] Mateo, J. L. and Fernández-Caballero, A. (2009). Finding out general tendencies in speckle noise reduction in ultrasound images. Expert Systems with Applications, 36(4):7786-7797. 
[Misra and Lim, 2015] Misra, A. and Lim, H. (2015). Nonlocal speckle denoising model based on non-linear partial differential equations. In Mandal, J. K., Satapathy, S. C., Kumar Sanyal, M., Sarkar, P. P., and Mukhopadhyay, A., editors, Information Systems Design and Intelligent Applications, volume 340 of Advances in Intelligent Systems and Computing, pages 165-176. Springer India.

[Naimi et al., 2015] Naimi, H., Adamou-Mitiche, A. B. H., and Mitiche, L. (2015). Medical image denoising using dual tree complex thresholding wavelet transform and wiener filter. Journal of King Saud University - Computer and Information Sciences, 27(1):40-45.

[Narayanan and Wahidabanu, 2009] Narayanan, S. K. and Wahidabanu, R. S. D. (2009). A view on despeckling in ultrasound imaging. International Journal of Signal Processing, Image Processing and Pattern Recognition, 2(3):85-98.

[Pantelic et al., 2007] Pantelic, R. S., Ericksson, G., and Hankamer, N. H. B. (2007). Bilateral edge filter: Photometrically weighted, discontinuity based edge detection. Journal of Structural Biology, 160(1):93-102.

[Peng et al., 2006] Peng, Q., Jones, R. C., and Constantinou, C. E. (2006). 2d ultrasound image processing in identifying responses of urogenital structures to pelvic floor muscle activity. Annals of biomedical engineering, 34(3):477-493.

[Perona and Malik, 1990] Perona, P. and Malik, J. (1990). Scale-space and edge detection using anisotropic diffusion. IEEE Transactions on Pattern Analysis and Machine Intelligence, 12(7):629639.

[Pizurica et al., 2003] Pizurica, A., Zlokolica, V., and Philips, W. (2003). Combined wavelet domain and temporal video denoising. In Proceedings of the IEEE Conference on Advanced Video and Signal Based Surveillance, AVSS ’03, pages 334-341. IEEE Computer Society.

[Raj and Venkateswarlu, 2012] Raj, V. N. P. and Venkateswarlu, T. (2012). Article:

Ultrasound medical image denoising using hybrid bilateral filtering. International Journal of Computer Applications, 56(14):44-51.

[Rudin et al., 1992] Rudin, L. I., Osher, S., and Fatemi, E. (1992). Nonlinear total variation based noise removal algorithms. Journal of Physica D, 60(1-4):259-268.

[Santos et al., 2013] Santos, A. M. F., dos Santos, R. M., Castro, P. M. A., Azevedo, E., Sousa, L., and Tavares, J. M. R. (2013). A novel automatic algorithm for the segmentation of the lumen of the carotid artery in ultrasound b-mode images. Expert Systems with Applications, 40(16):6570-6579. 
[Sattar et al., 1997] Sattar, F., Floreby, L., Salomonsson, G., and Lovstrom, B. (1997). Image enhancement based on a nonlinear multiscale method. IEEE Transactions on Image Processing, 6(6):888-895.

[Shao et al., 2013] Shao, D., Liu, P., and Liu, D. C. (2013). Characteristic matching-based adaptive fast bilateral filter for ultrasound speckle reduction. Pattern Recognition Letters, 34(5):463-469.

[Sheikh and Bovik, 2006] Sheikh, H. and Bovik, A. (2006). Image information and visual quality. IEEE Transactions on Image Processing, 15(2):430-444.

[Thaipanich et al., 2010] Thaipanich, T., Oh, B. T., Wu, P.-H., Xu, D., and Kuo, C. C. (2010). Improved image denoising with adaptive nonlocal means (anl-means) algorithm. IEEE Transactions on Consumer Electronics, 56(4):2623-2630.

[Tomasi and Manduchi, 1998] Tomasi, C. and Manduchi, R. (1998). Bilateral filtering for gray and color images. In Proceedings of the Sixth International Conference on Computer Vision, ICCV '98, pages 839-847, Washington, DC, USA. IEEE Computer Society.

[Verma et al., 2015] Verma, K., Singh, B. K., and Thoke, A. (2015). An enhancement in adaptive median filter for edge preservation. Procedia Computer Science, 48:29-36. International Conference on Computer, Communication and Convergence (ICCC 2015).

[Wang and Bovik, 2002] Wang, Z. and Bovik, A. (2002). A universal image quality index. IEEE Signal Processing Letters, 9(3):81-84.

[Wang et al., 2004] Wang, Z., Bovik, A. C., Sheikh, H. R., and Simoncelli, E. P. (2004). Image quality assessment: from error visibility to structural similarity. IEEE Transactions on Image Processing, 13(4):600-612.

[Wu et al., 2010] Wu, Y.-H., Lo, Y.-C., Cheng, S.-C., and Lin, C.-L. (2010). Adaptive ultrasound image matching for automatic liver disease diagnosis using generalized Hough transform. In Proceedings of the 2010 Sixth International Conference on Intelligent Information Hiding and Multimedia Signal Processing, IIH-MSP '10, pages 627-630, Washington, DC, USA. IEEE Computer Society.

[Yang et al., 2011] Yang, H.-Y., Wang, X.-Y., Qu, T.-X., and Fu, Z.-K. (2011). Image denoising using bilateral filter and Gaussian scale mixtures in shiftable complex directional pyramid domain. Computer and Electrical Engineering, 37(5):656-668. 
[Yang et al., 2015] Yang, K., Zhao, Y., and Deng, N. (2015). Fast bilateral filtering using the discrete cosine transform and the recursive method. Optik - International Journal for Light and Electron Optics, 126(6):592-595.

[Yu and Acton, 2002]Yu, Y. and Acton, S. T. (2002). Speckle reducing anisotropic diffusion. IEEE Transactionson Image Processing, 11(11):260-270.

[Zhang and Allebach, 2008] Zhang, B. and Allebach, J. P. (2008). Adaptive bilateral filter for sharpness enhancement and noise removal. IEEE Transactions on Image Processing, 17(5):664678.

[Zhang and Wang, 2015] Zhang, C. and Wang, K. (2015). A switching median-mean filter for removal of high-density impulse noise from digital images. Optik - International Journal for Light and Electron Optics, 126(9-10):956-961. 


\section{FIGURE CAPTION}

Figure 1: Flowchart of the proposed smoothing method.

Figure 2: Original synthetic image, after being corrupted by artificial speckle noise with variance equal to 0.3 , and results of the smoothing achieved by the methods under comparison: (A) median filter, (B) average filter, (C) Perona and Malik Anisotropic diffusion filter, (D) proposed selective average filter, (E) DPAD method, (F) 2D Bilateral filter, (G) Frost filter and (H) Wiener filter.

Figure 3: Values obtained for the PSNR, EPI, VIF, MSSIM and IQI metrics for the images corrupted with artificial speckle noise smoothed by the filters under comparison (Figure 2).

Figure 4: Original synthetic image, after being submitted to the Field II ultrasound simulator software, and results of the smoothing achieved by the methods under comparison: (A) median filter, (B) average filter, (C) Perona and Malik Anisotropic diffusion filter, (D) proposed selective average filter, (E) DPAD method, (F) 2D Bilateral filter, (G) Frost filter and (H) Wiener filter.

Figure 5: Values obtained for the PSNR, EPI, VIF, MSSIM and IQI metrics for the images submitted to the Field II ultrasound simulator software smoothed by the filters under comparison (Figure 4).

Figure 6: Results of the smoothing methods under comparison from a synthetic image corrupted with artificial speckle noise: (A) median filter, (B) average filter, (C) Perona and Malik Anisotropic diffusion filter, (D) proposed selective average filter, (E) DPAD method, (F) 2D Bilateral filter, (G) Frost filter and $(\mathrm{H})$ Wiener filter. 
Figure 7: Average values obtained for the PSNR, EPI, VIF, MSSIM and IQI metrics for all synthetic images smoothed by the filters under comparison.

Figure 8: From left to right, example of three ultrasound images of the pelvic cavity and one ultrasound image of the carotid artery used to compare the smoothing methods under comparison.

Figure 9: Results of the smoothing methods under comparison from the real ultrasound images in Figure 8: (A) median filter, (B) average filter, (C) Perona and Malik Anisotropic diffusion filter, (D) proposed selective average filter, (E) DPAD method, (F) 2D Bilateral filter, (G) Frost filtering and (H) Wiener filter.

Figure 10: Segmentation results from a region of the urethra selected from two real ultrasound images that were or not smoothed by the proposed method. 


\section{TABLE CAPTION}

Table 1: ENL computed from the synthetic images in Figures 2 and 4. (Bold values are the best values obtained, which indicate a smoothing of superior quality.)

Table 2: ENL computed from the real ultrasound images in Figures 9. (Bold values are the best values obtained, which indicate a smoothing of superior quality.) 
TABLES

Table 1

\begin{tabular}{|c|c|c|c|c|c|c|c|c|c|}
\hline$\#$ & $\begin{array}{c}\text { Simulated } \\
\text { image }\end{array}$ & $\begin{array}{c}\text { Median } \\
\text { filter }\end{array}$ & $\begin{array}{c}\text { Average } \\
\text { filter }\end{array}$ & $\begin{array}{c}\text { Anisotropic } \\
\text { Diffusion } \\
\text { filter }\end{array}$ & DPAD & $\begin{array}{c}2 \mathrm{D} \\
\text { Bilateral } \\
\text { filter }\end{array}$ & $\begin{array}{c}\text { Frost } \\
\text { filter }\end{array}$ & $\begin{array}{c}\text { Wiener } \\
\text { filter }\end{array}$ & $\begin{array}{c}\text { Proposed } \\
\text { method }\end{array}$ \\
\hline Figure 2 & 5.9925 & 26.9146 & 54.1989 & 250.5487 & 59.3685 & 8.1167 & 135.7024 & 146.8080 & $\mathbf{2 5 1 . 7 7 2 0}$ \\
\hline Figure 4 & 24.1555 & 52.2514 & 60.2226 & $\mathbf{2 9 1 . 6 7 3 2}$ & 137.9077 & 65.2842 & 107.6297 & 113.4500 & 173.3482 \\
\hline
\end{tabular}

\section{Table 2}

\begin{tabular}{|c|c|c|c|c|c|c|c|c|c|}
\hline$\#$ & $\begin{array}{c}\text { Simulated } \\
\text { image }\end{array}$ & $\begin{array}{c}\text { Median } \\
\text { filter }\end{array}$ & $\begin{array}{c}\text { Average } \\
\text { filter }\end{array}$ & $\begin{array}{c}\text { Anisotropic } \\
\text { Diffusion } \\
\text { filter }\end{array}$ & DPAD & $\begin{array}{c}\text { 2D } \\
\text { Bilateral } \\
\text { filter }\end{array}$ & $\begin{array}{c}\text { Frost } \\
\text { filter }\end{array}$ & $\begin{array}{c}\text { Wiener } \\
\text { filter }\end{array}$ & $\begin{array}{c}\text { Proposed } \\
\text { method }\end{array}$ \\
\hline $\begin{array}{c}\text { Figure 9 - } \\
\text { Seq. 1 }\end{array}$ & 30.2769 & 67.3820 & 91.5440 & 133.4084 & 150.4175 & 62.2093 & 121.7063 & 92.2860 & $\mathbf{4 1 5 . 8 8 4 0}$ \\
\hline $\begin{array}{c}\text { Average } \\
\text { for seq. 1 }\end{array}$ & 24.5568 & 37.5436 & 42.2675 & 52.9957 & 33.6917 & 38.8445 & 48.5982 & 45.5038 & $\mathbf{8 0 . 9 1 2 0}$ \\
\hline $\begin{array}{c}\text { Figure 9 - } \\
\text { seq. 2 }\end{array}$ & 12.1627 & 21.2455 & 23.4751 & 46.5803 & 55.8347 & 27.8882 & 31.7100 & 32.8159 & $\mathbf{6 0 . 0 5 1 5}$ \\
\hline $\begin{array}{c}\text { Average } \\
\text { for seq. 2 }\end{array}$ & 16.7982 & 28.3208 & 31.0591 & 62.9522 & 19.3905 & 46.6149 & 41.4040 & 42.0562 & $\mathbf{8 5 . 7 8 0 6}$ \\
\hline $\begin{array}{c}\text { Figure 9 - } \\
\text { seq. 3 }\end{array}$ & 27.2426 & 46.2159 & 50.4132 & 86.4907 & 147.6368 & 55.2607 & 62.8385 & 62.1842 & $\mathbf{3 9 7 . 1 1 9 0}$ \\
\hline $\begin{array}{c}\text { Average } \\
\text { for seq. 3 }\end{array}$ & 19.6943 & 29.7707 & 32.2397 & 50.3927 & 25.2270 & 33.6893 & 38.7927 & 38.3110 & $\mathbf{1 4 7 . 4 2 5 9}$ \\
\hline $\begin{array}{c}\text { Figure 9 - } \\
\text { seq. 4 }\end{array}$ & 16.2348 & 16.8593 & 17.8152 & 23.8995 & 16.4467 & 22.4444 & 20.0212 & 19.0357 & $\mathbf{3 0 . 8 8 2 3}$ \\
\hline $\begin{array}{c}\text { Average } \\
\text { for seq. 4 }\end{array}$ & 18.1653 & 18.8240 & 19.6801 & 26.5389 & 18.8888 & 23.6422 & 21.7645 & 20.4499 & $\mathbf{3 7 . 8 6 3 0}$ \\
\hline
\end{tabular}




\section{FIGURES}

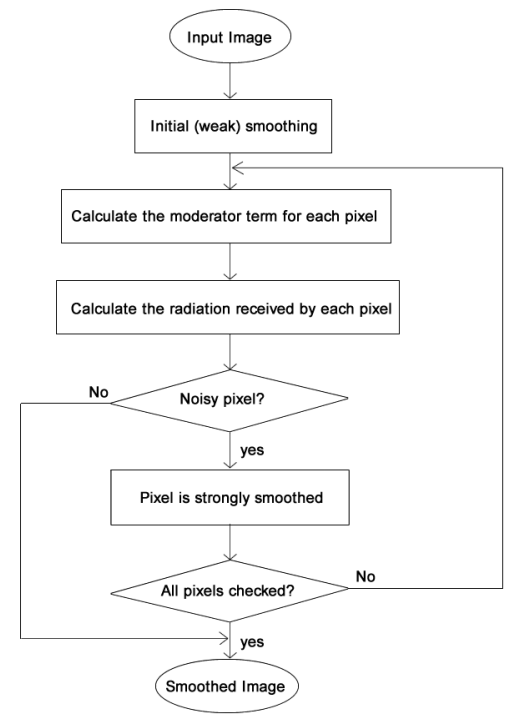

Figure 1 


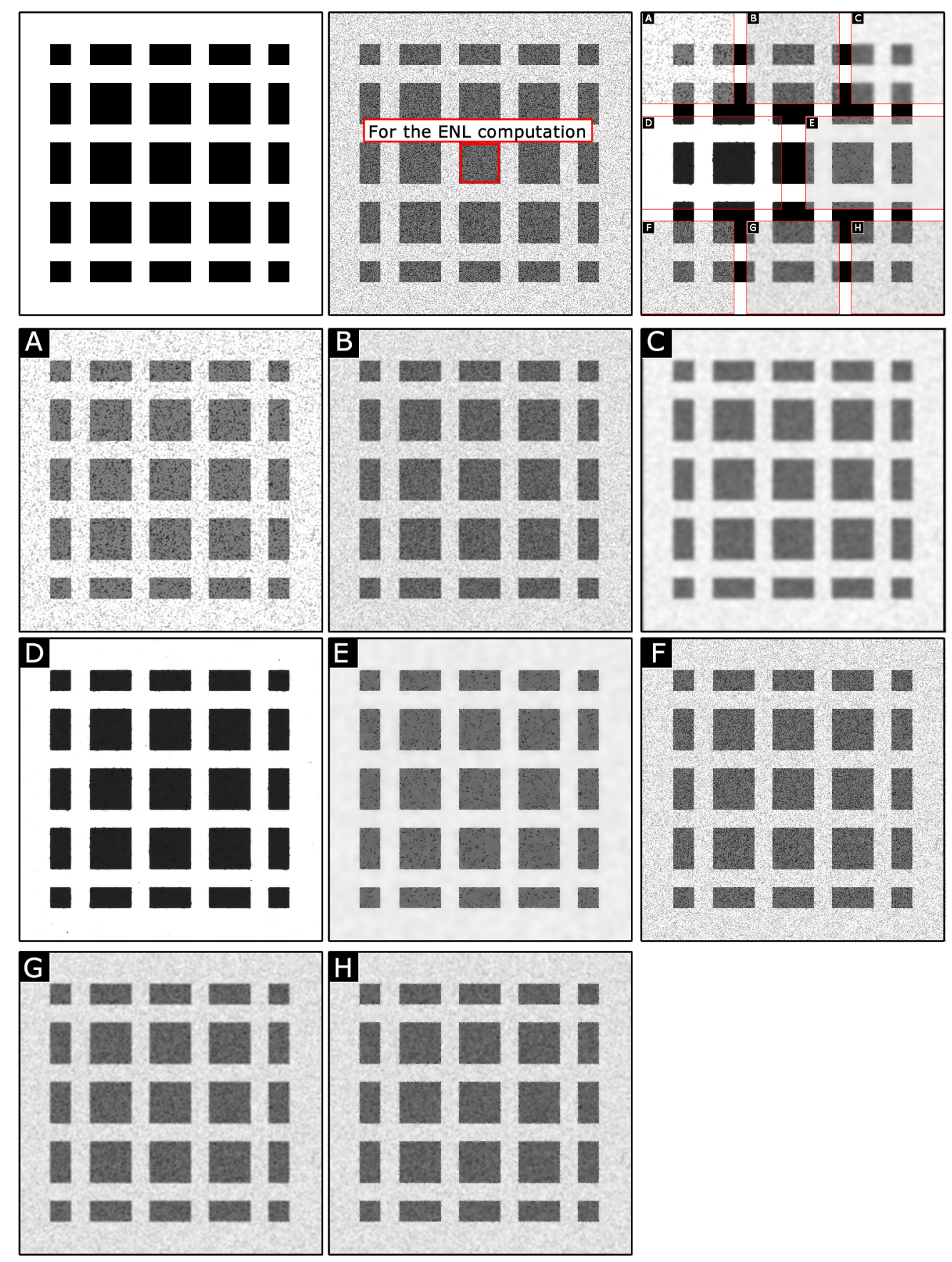

Figure 2 


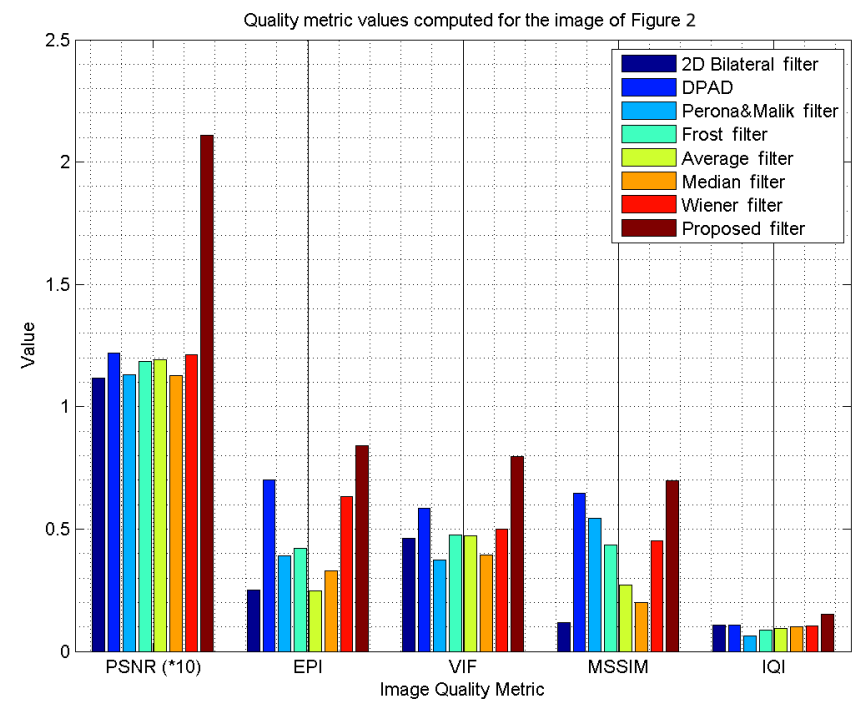

Figure 3
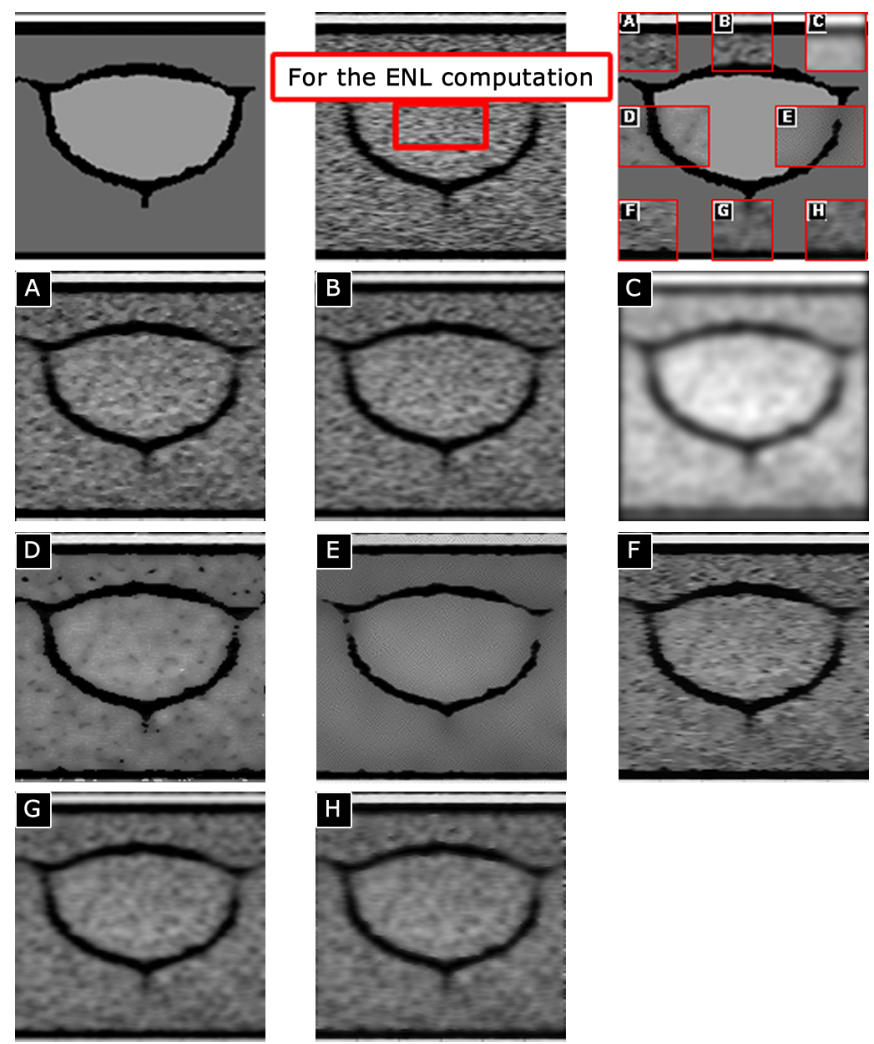

Figure 4 


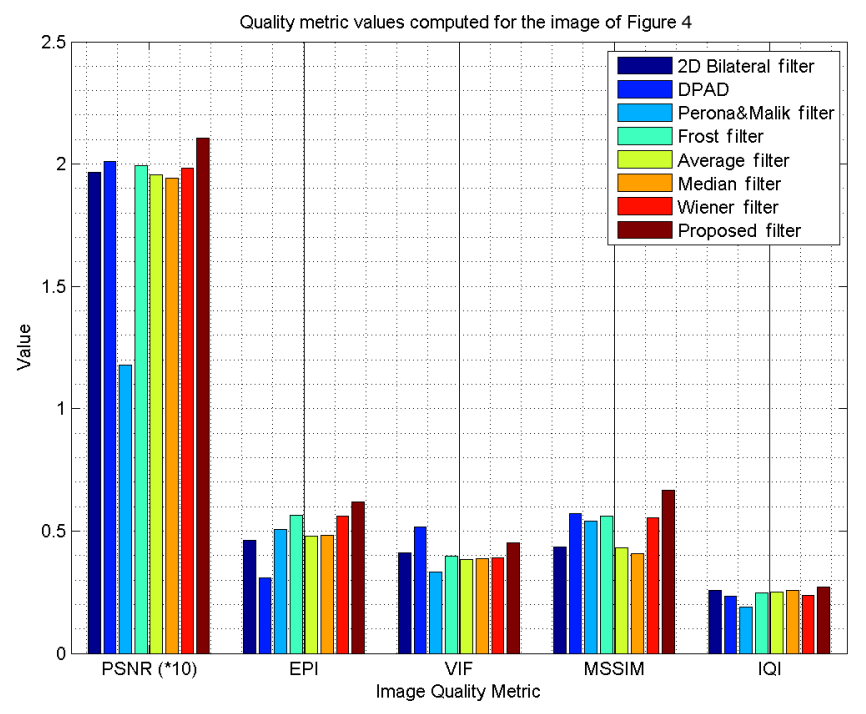

Figure 5

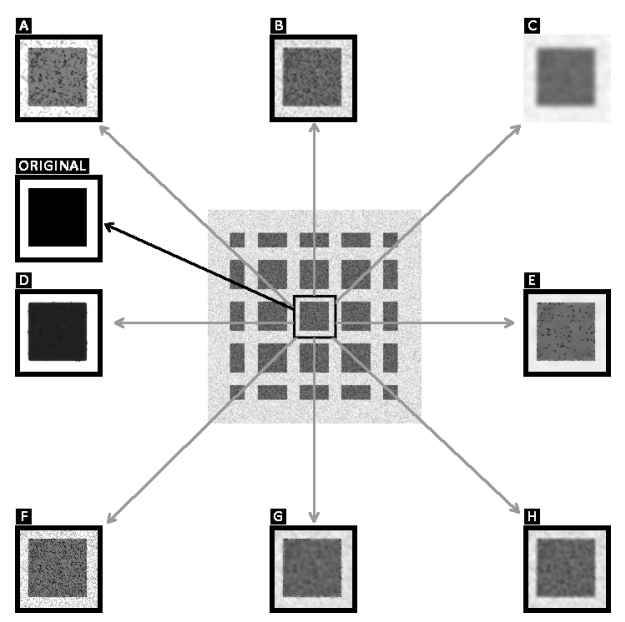

Figure 6

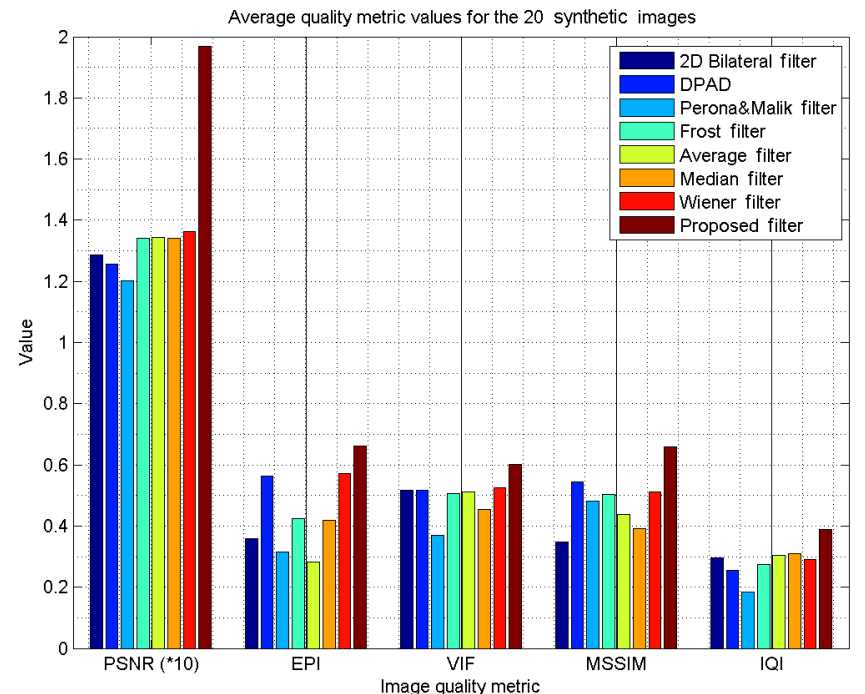

Figure 7 

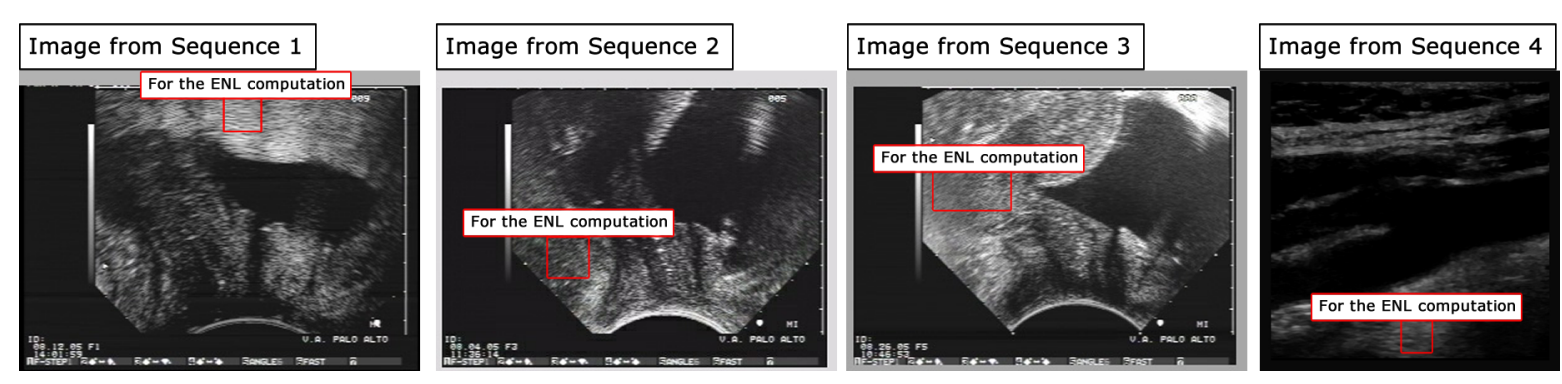

Figure 8
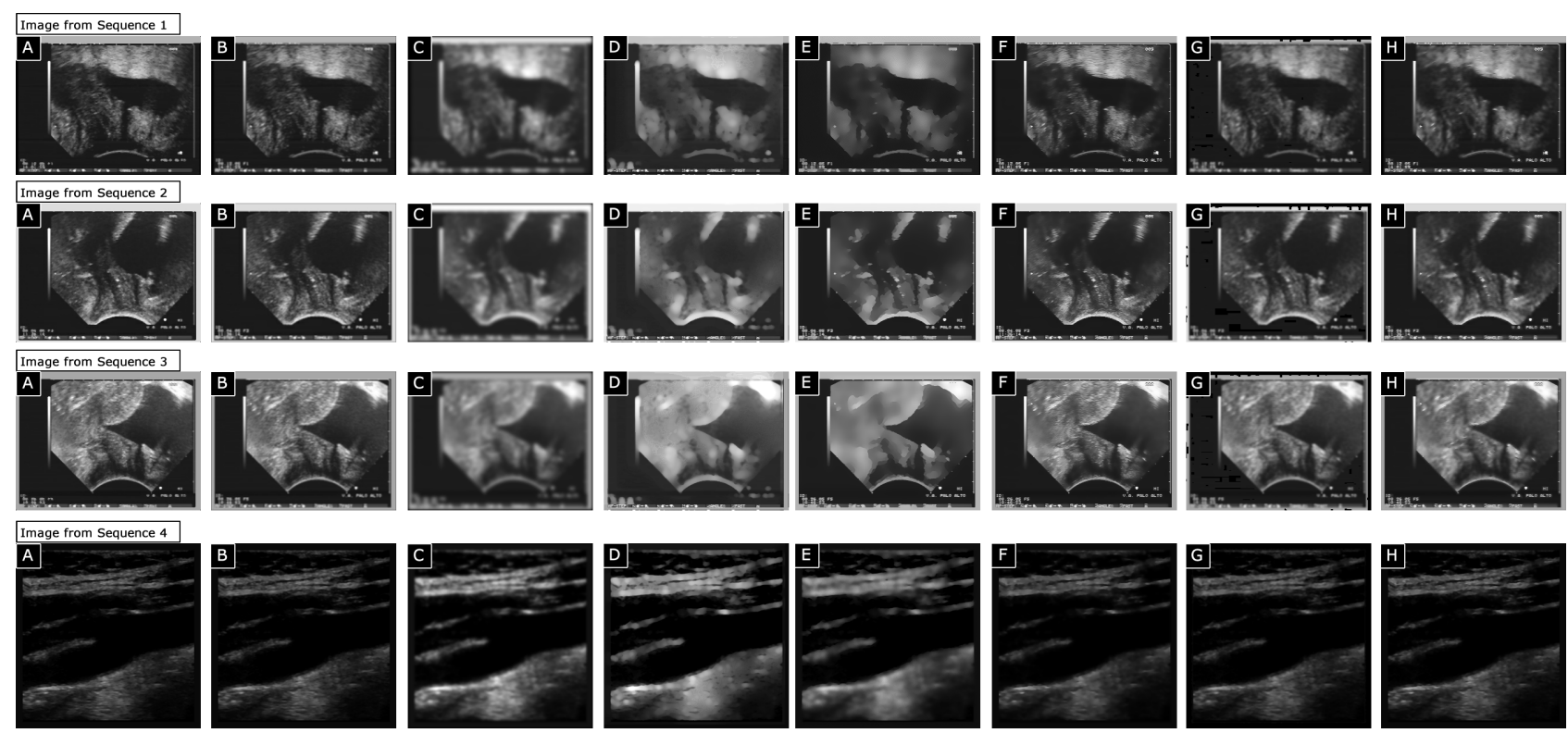

Figure 9

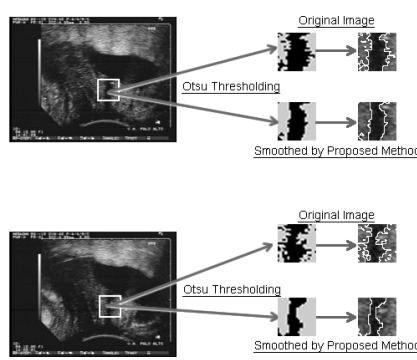

Figure 10 